\title{
Barley NARROW LEAFED DWARF1 encoding a WUSCHEL-RELATED HOMEOBOX 3 (WOX3) regulates the marginal development of lateral organs
}

\author{
Takanori Yoshikawa*1), Shin-Ya Tanaka $^{1)}$, Yuuki Masumoto' ${ }^{1)}$, Naoya Nobori' ${ }^{1)}$, Hiroto Ishii ${ }^{1)}$, \\ Ken-Ichiro Hibara ${ }^{2)}$, Jun-Ichi Itoh ${ }^{2)}$, Takatoshi Tanisaka ${ }^{1)}$ and Shin Taketa ${ }^{3)}$ \\ 1) School of Agricultural Regional Vitalization, Kibi International University, Minamiawaji, Hyogo 656-0484, Japan \\ 2) Graduate School of Agricultural and Life Sciences, University of Tokyo, Tokyo 113-8657, Japan \\ 3) Group of Genetic Resources and Functions, Institute of Plant Science and Resources, Okayama University, Kurashiki, Okayama 710- \\ 0046, Japan
}

\begin{abstract}
Barley (Hordeum vulgare L.) is the fourth most-produced cereal in the world and is mainly utilized as animal feed and malts. Recently barley attracts considerable attentions as healthy food rich in dietary fiber. However, limited knowledge is available about developmental aspects of barley leaves. In the present study, we investigated barley narrow leafed dwarfl (nld1) mutants, which exhibit thin leaves accompanied by short stature. Detailed histological analysis revealed that leaf marginal tissues, such as sawtooth hairs and sclerenchymatous cells, were lacked in $n l d l$, suggesting that narrowed leaf of $n l d l$ was attributable to the defective development of the marginal regions in the leaves. The defective marginal developments were also appeared in internodes and glumes in spikelets. Map-based cloning revealed that $N L D 1$ encodes a WUSCHEL-RELATED HOMEOBOX 3 (WOX3), an ortholog of the maize NARROW SHEATH genes. In situ hybridization showed that NLD1 transcripts were localized in the marginal edges of leaf primordia from the initiating stage. From these results, we concluded that $N L D 1$ plays pivotal role in the increase of organ width and in the development of marginal tissues in lateral organs in barley.
\end{abstract}

Key Words: barley, Hordeum vulgare L., narrow leafed dwarf1, WUSCHEL-RELATED HOMEOBOX 3, marginal development, lateral organ.

\section{Introduction}

Leaves are the major photosynthetic organs in plants. The light-capture efficiency significantly differs depending on the leaf shapes, angles and arrangements. Thus, leaf morphology is critical for the survival of plant species. After the cell fate is determined in the shoot apical meristems (SAMs), leaf primordia grow in accordance with three axes; the proximal-distal, adaxial-abaxial, and medial-lateral directions (Moon and Hake 2011, Scarpella et al. 2010). The accurate developments along these axes ensure the morphogenesis of sophisticated leaf organs with high reproducibility.

The plant hormone auxin plays pivotal roles in the leaf development (Benjamins and Scheres 2008). Auxin is unique in its polar transportation due to the localized influx carriers and efflux carriers (Petrášek and Friml 2009). Once transported to SAMs, auxin flows to the leaf-primordium

Communicated by T. Komatsuda

Received February 4, 2016. Accepted March 7, 2016.

First Published Online in J-STAGE on May 20, 2016.

*Corresponding author (e-mail: t-yoshi@kiui.ac.jp) initiation sites through the epidermis layer L1 mediated by PIN-FROMED 1 (Petrášek and Friml 2009, Scarpella et al. 2010). Such auxin localization down-regulate class $I$ KNOTTED1-like homeobox (KNOX) genes and promote the outgrowth of primordia, creating leaf tips, and the basipetal streams of auxin from the tip through the internal tissue induce the differentiation of vascular strand (Hay et al. 2004, Scarpella et al. 2006, Wenzel et al. 2007). Interestingly, similar auxin-mediated mechanisms also control the development of leaflet in the compound leaf (Barkoulas et al. 2008, Giacomo et al. 2013) and leaf serrations in Arabidopsis thaliana (Aloni et al. 2003, Bilsborough et al. 2011, Hay et al. 2006).

Soon after the initiation, the regions of leaf primordia facing the SAMs or away from the SAMs aquire the identity as the adaxial or abaxial side, respectively. Through the loss-of-function and/or gain-of-function analyses, the involvement of many genes in the establishment of adaxialabaxial polarity has been revealed; in the case of $A$. thaliana, adaxial identity is regulated by class III HOMEODOMAINLEUCINE ZIPPER family genes and ASYMMETRIC LEAVES2 while abaxial identity is regulated by $Y A B B Y$ 
family genes, KANADI family genes, and $A U X I N$ RESPONSE FACTOR family genes (Nakata and Okada 2013). The adaxial or abaxial specific expression of these genes is crucial for the establishment of the organ polarity, and adaxial/abaxial regulators are interacting antagonistically to maintain the expression regions (Nakata and Okada 2013, Scarpella et al. 2010). Other than gene interactions, small RNAs and auxin localization are also crucial for the establishment of the organ polarity (Heisler et al. 2005, Nakata and Okada 2013, Vernoux et al. 2010). The loss of adaxialabaxial polarity induces the formation of narrow or needle leaves in A. thaliana (Sarojam et al. 2010, Stahle et al. 2009), suggesting that lamina growth is regulated downstream of the adaxial-abaxial polarity.

Many genetic approaches have been employed to reveal the lamina growth mechanisms, demonstrating that WUSCHELRELATED HOMEOBOX (WOX) genes are critical for the development of leaf lateral domains. In maize, the loss-offunction mutations in both NARROW SHEATH1 (NS1) and $N S 2$, which encode the duplicated $W O X 3$ genes, result in the lack of marginal regions in leaves and floral organs as well as the shortened internode on the marginal side of the stem (Nardmann et al. 2004, Scanlon et al. 1996, Scanlon and Freeling 1998). The NS transcripts are accumulated in the marginal edges of leaf primordia, and $n s 1$ ns 2 double mutants fail to down-regulate KNOX proteins in the premarginal regions of leaf primordia, leading to the deletion of marginal region from the primordial stages (Nardmann et al. 2004, Scanlon et al. 1996). These results suggest that NS genes play pivotal roles in the recruitment of leaf foundercells by down-regulating KNOX accumulation although the mechanism is still unclear (Scanlon 2000, Scanlon and Freeling 1997, Scanlon et al. 2000). Similar developmental defects in lateral domains were observed in the $N S$ orthologue mutants in rice (NARROW LEAF 2 [NAL2], NAL3; Cho et al. 2013, Ishiwata et al. 2013) and A. thaliana (PRESSED FLOWER1 [PRS1]; Matsumoto and Okada 2001, Nardmann et al. 2004), suggesting the conserved function of NS-related genes in the development of lateral organs.

WOX1 also plays a central role in lamina development. $W O X 1$ is unique in that it belongs to the same clade of the $W O X 3 / P R S$ family but seems to be absent in grasses (Haecker et al. 2004, Vandenbussche et al. 2009). The lossof-function of WOX1 lead to severe defects in lamina outgrowth in petunia (MAEWEST; Vandenbussche et al. 2009), tobacco (LAMI; McHale and Marcotrigiano 1998), and Medicago truncatula (STENOFOLIA; Tadege et al. 2011). $W O X 1$ genes are expressed in the middle mesophyll layers and at the leaf margin cells, similar to WOX3/PRS expression patterns, and woxl prs double mutants exhibit not only the lost of leaf marginal tissues but also the confused adaxial-abaxial identity at leaf margin regions (Nakata et al. 2012, Vandenbussche et al. 2009). These results suggest that $W O X 1$ and $W O X 3 / P R S$ play pivotal role not only in lamina outgrowth but also in the formation of adaxial- abaxial boundaries at leaf margins.

Barley (Hordeum vulgare L.) is the fourth mostproduced cereal in the world and is mainly utilized as animal feed and malts. Recently, barley attracts considerable attentions as healthy food rich in dietary fiber. The diploid nature makes barley a model crop of Triticeae. However, limited knowledge is available about developmental aspects of barley leaves. In the present study, we investigated barley narrow leafed dwarf1 (nld1) mutants whose phenotypes are thinner leaves accompanied by short stature. Detailed histological analysis indicated that narrowed leaf of $n l d l$ was attributable to the lack of marginal regions. Map-based cloning revealed that $N L D 1$ encodes a maize NS-related WOX3 protein, and we also found the marginal expression of NLD1. The results presented in this study indicate that $N L D 1$ plays pivotal role in the increase of organ width and in the development of marginal tissues in lateral organs in barley.

\section{Materials and Methods}

\section{Plant materials}

In the present study, we used two alleles of NLD1: nld1.a and nld1.b. The nld1.a was first isolated as a spontaneous mutant "Nagaoka Dwarf" from the $\mathrm{F}_{2}$ population of the cross Nagaoka $\times$ Marumi 16 (Takahashi et al. 1972), however, it was phenotypically quite similar to Nagaoka. Thus, this mutant was named as Nagaoka-dwarf, and its original cultivar was designated as Nagaoka here. Another independent mutant, $n l d 1 . b$ is a gamma-ray induced mutant derived from a line Kanto Nijo 29 (KN29). For the evaluation of mutant phenotypes, mutants and wild-type seeds were sown on soil and grown under natural conditions. To promote germination, seeds were kept at $15^{\circ} \mathrm{C}$ on wet paper for three days before sowing.

\section{Epidermal cell observation}

The second leaf blades of $n l d l$ and wild-type were fixed with FAA (formaldehyde:glacial acetic acid:50\% ethanol [2:1:17]) for $24 \mathrm{~h}$ at $4^{\circ} \mathrm{C}$. They were then dehydrated in a graded ethanol series. Dehydrated samples were incubated at $96^{\circ} \mathrm{C}$ in chloralhydrate dissolved in $100 \%$ ethanol until they were cleared, and observed with a light microscope. The measurement of cell width was performed by image analysis with Image J (available at http://rsbweb.nih.gov/ij/).

\section{Paraffin sectioning and histological analysis}

Plant samples of $n l d 1$ and wild-type were fixed with FAA (formaldehyde:glacial acetic acid:50\% ethanol [2:1:17]) for $24 \mathrm{~h}$ at $4{ }^{\circ} \mathrm{C}$ for histological analysis, or fixed with PFA (4\% $(\mathrm{w} / \mathrm{v})$ paraformaldehyde and $1 \%$ Triton $\mathrm{X}$ in $0.1 \mathrm{M}$ sodium phosphate buffer) for $48 \mathrm{~h}$ at $4^{\circ} \mathrm{C}$ for in situ hybridization. They were then dehydrated in a graded ethanol series, substituted with 1-butanol, and embedded in Paraplast ${ }^{\circledR}$ Plus (McCormick Scientific). The samples were sectioned at $8 \mu \mathrm{m}$ thick using a rotary microtome. For the histological 
analysis, sections were stained in haematoxylin or doublestained in safranin and fast green. After staining, sections were mounted with Poly-Mount ${ }^{\circledR}$ (Polysciences, Inc.) and observed with a light microscope. The measurement of leaf primordium width was performed by image analysis with Image J (available at http://rsbweb.nih.gov/ij/).

\section{In situ hubridization}

Paraffin sections were prepared as mentioned above. Digoxigenin-labeled anti-sense and sense RNA probes were prepared from a 666-bp fragment of $N L D 1$, which was amplified by PCR with forward primer (5'-AGCAGCTGATG ATCCTGGAG- $3^{\prime}$ ) and reverse primer (5'-AGGTGGAGCA AGAGGAGGAC- $3^{\prime}$ ) using cDNA as a template. The amplified PCR product was cloned into $\mathrm{pCR}^{\mathrm{TM}}$-Blunt vector (invitrogen), followed by in vitro transcription using DIG RNA Labeling Kit (Roche). In situ hybridization and immunological detection with alkaline phosphatase were performed according to the methods of Kouchi and Hata (1993).

\section{Map-based cloning}

For the fine mapping of $N L D 1$ gene, nld1.b plants were crossed with normal barley (NC117), and $105 \mathrm{~F}_{2}$ plants were used for mapping. The previous study reported that $N L D 1$ is located about $21.6 \mathrm{cM}$ proximal from the fragile stem 1 locus on chromosome 5HL (Hayashi et al. 1983, Takahashi et al. 1972). Thus, genotyping were performed using five markers (k09239, k03390, k01939, k04066, Bmag0337) (Sato et al. 2009, Varshney et al. 2007), which locate in the vicinity of predicted NLD1 locus, and the candidate region was further limited from $\mathrm{k} 03390(76.4 \mathrm{cM})$ to k01939 (80.7 cM). Since this region include WOX3 encoding $M L O C$ 7772.1, an orthologous gene of maize NS1 and $N S 2$ and rice $N A L 2 / 3$, we compared the genomic sequence of the gene between $n l d 1$ mutants and wild-types.

\section{Results}

\section{Phenotypes of nld1 mutants in the vegetative phase}

In the present study, we used two alleles of NLD1: nld1.a and nld1.b (see Materials and Methods). The nld1.a is a spontaneous mutant isolated from a six-rowed cultivar Nagaoka, and $n l d 1 . b$ is a gamma-ray induced mutant of a two-rowed line Kanto Nijo 29 (KN29). Thus, Nagaoka and $\mathrm{KN} 29$, both of which are covered caryopsis types, were used as a wild-type of nld1.a and nld1.b, respectively, in all experiments. While the leaf blade lengths of nld1 mutants were comparable to those of wild-types, the leaf blade widths were clearly reduced to about $60 \%$ of those of wildtypes (Fig. 1a-1e). These narrow leaf phenotypes were constantly observed through all leaves; from the first leaf until the matured adult leaves (Fig. 1e), and the leaf emerging rate in $n l d 1$ mutants were comparable to those of wildtypes (Supplemental Fig. 1). To reveal the cause for the reduction in leaf blade width, we compared the width and the number of epidermal cells in leaf blade between wild-type
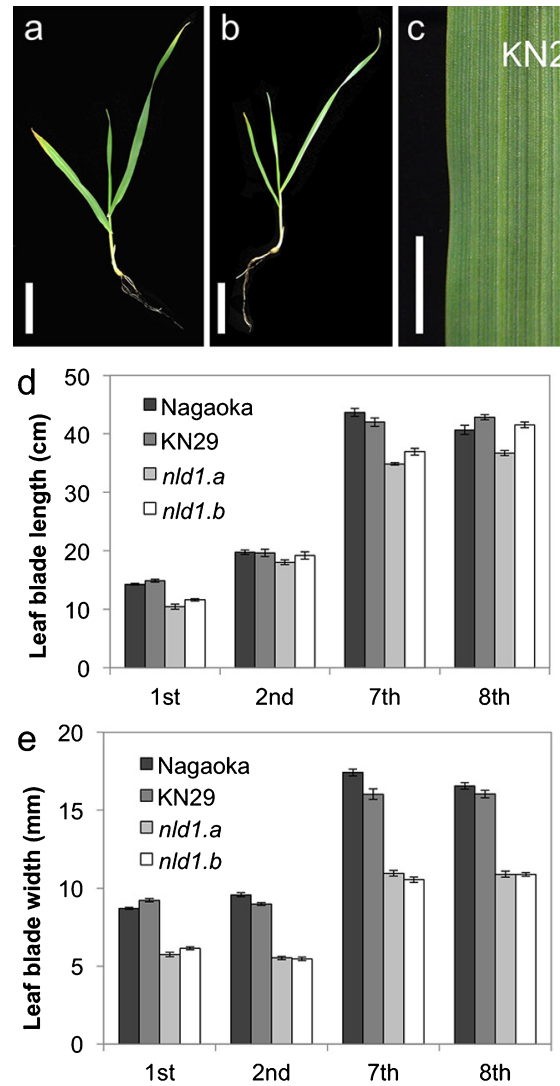
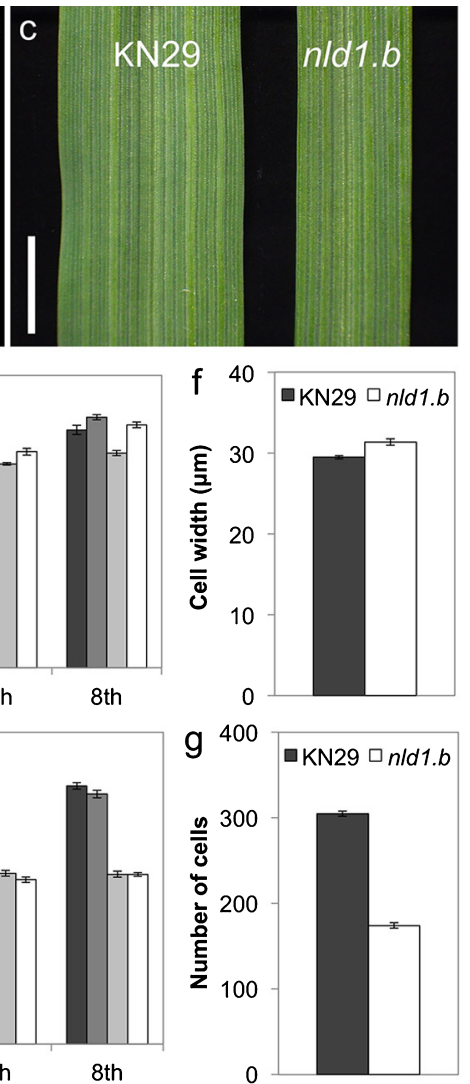

Fig. 1. Leaf blade phenotypes of wild-type and nld1 mutants. The nld1. $a$ is a spontaneous mutant isolated from a cultivar Nagaoka, and $n l d 1 . b$ is a gamma-ray induced mutant derived from a line Kanto Nijo 29 (KN29). Thus, Nagaoka and KN29 were used as a wild-type of nld1. $a$ and nld1.b, respectively, in all experiments (see Materials and Methods). ( $a$ and $b$ ) Seedlings at the second leaf stage of wild-type (KN29) (a) and $n l d 1 . b$ (b). (c) Leaf blades of the second leaf in wild-type (KN29) and nld1.b. (d and e) Comparison of leaf blade length (d) and width (e) between wild-type (Nagaoka and KN29) and nld1 mutants. ( $f$ and $g$ ) Comparison of cell width ( $f$ ) and number of cells $(g)$ in the second leaf blades between wild-type (KN29) and nld1.b. Results are shown as means $\pm \operatorname{SE}(\mathrm{n}=10)(\mathrm{d}, \mathrm{e}, \mathrm{f}, \mathrm{g})$. Bars $=5 \mathrm{~cm}(\mathrm{a}, \mathrm{b}), 5 \mathrm{~mm}(\mathrm{c})$.

and $n l d 1$ mutant. While the width of the epidermal cells in nld 1 was comparable to those of wild-type, the number of cells was about $57 \%$ of those of wild-type (Fig. 1f, 1g), indicating that the narrow leaf phenotype of $n l d l$ was attributable to the reduced cell number across leaf blade.

For the further analysis of leaf development, we performed histological analysis of leaf primordia in nld1 (Fig. 2a, 2b). In barley, leaf ridge development precedes leaf primordium development (Shewry 1992), and it is quite difficult to recognize the differentiation from leaf ridge to leaf primordium. Thus, in the present study, we defined the 6th leaf primordium as the P1 leaf primordium (the youngest primordial stage) in the 2nd leaf stage seedling (Supplemental Fig. 2). The comparison of leaf primordium width showed that $n l d 1$ already exhibited narrowed leaf phenotype at P1 primordial stage, and this trend was enhanced from P3 to P4 primordial stage (Fig. 2c, 2d). These results 

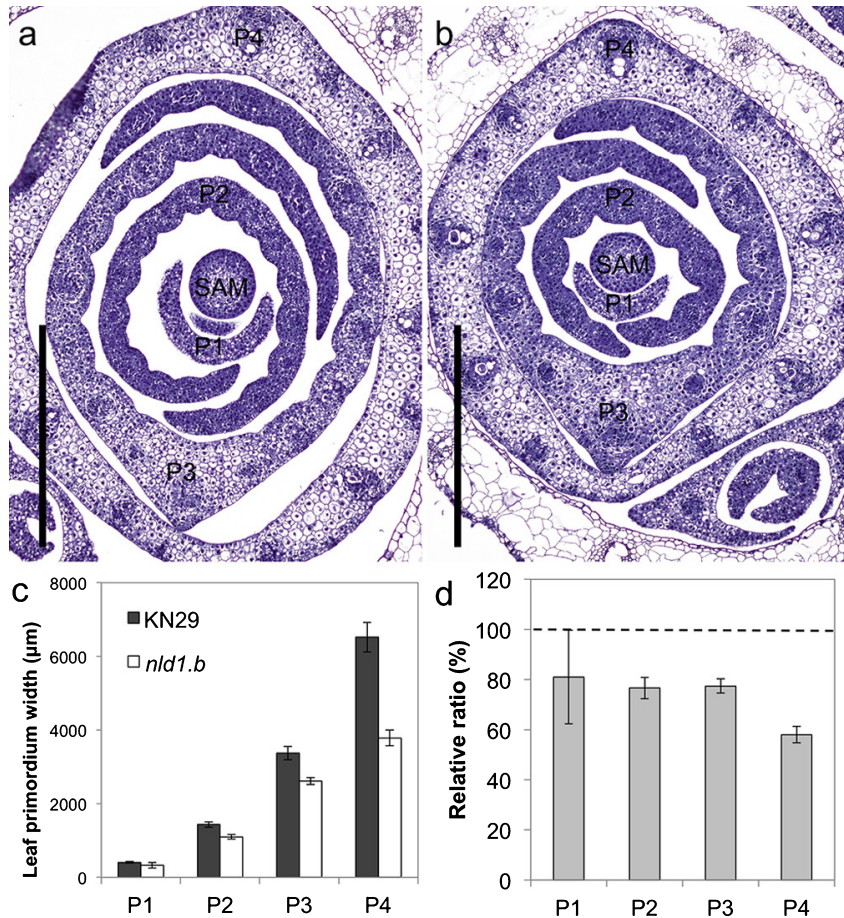

Fig. 2. Development of leaf primordia in wild-type and nld1.b. (a and b) Cross sections of shoot apexes in wild-type (KN29) (a) and nld1.b (b). Seedlings at the 2nd leaf stage are used as the plant materials. Shoot apical meristem (SAM) and leaf primordial stages (P1-P4) are shown in the figures. (c) Comparison of leaf-primordium width between wild-type (KN29) and nld1.b. (d) The relative ratio of leaf-primordium width in $n l d 1 . b$ by taking those of wild-type (KN29) as 100 . Results are shown as means $\pm \operatorname{SE}(n=5)(c, d)$. Bars $=500 \mu \mathrm{m}$ $(\mathrm{a}, \mathrm{b})$.

suggest that the narrowed leaf phenotype of $n l d 1$ is attributable to the impaired development of leaf primordium.

The $n l d 1$ mutants exhibit not only the reduction in leafblade width but also the obvious lack of the auricles in the lamina-joint (Fig. 3a-3d). Since auricles are formed in the margins of lamina-joint, these lacks in $n l d l$ indicate the possibility that $n l d l$ lack the marginal region in the leaf. The histological analysis of the leaf primordium also showed the abnormal development of the marginal region (Fig. 3e, 3f). Occasionally, nld1 shows asymmetrical development of the leaf in medial-lateral directions (Supplemental Fig. 3). Detailed analysis of the leaf margins revealed the defective development of the sawtooth hairs and sclerenchymatous cells together with the thickening of leaf edges in nld1 mutants (Fig. 3g-3j). Thus, it was indicated that nldl lack the marginal region of the leaf, which resulted in the significant reduction in leaf width. Interestingly, trichome developments were also impaired not only in the leaf edges but also in the surface of leaf-blades in mutants (Supplemental Fig. 4). The density of trichome, which is formed along with the longitudinal veins, is reduced in nld1 (Supplemental Fig. $4 a-4 c)$, and each trichome was smaller than those of wild-type (Supplemental Fig. 4d-4g). Occationary, quite narrow veins were formed in the leaf margins of nld $1 \mathrm{mu}-$

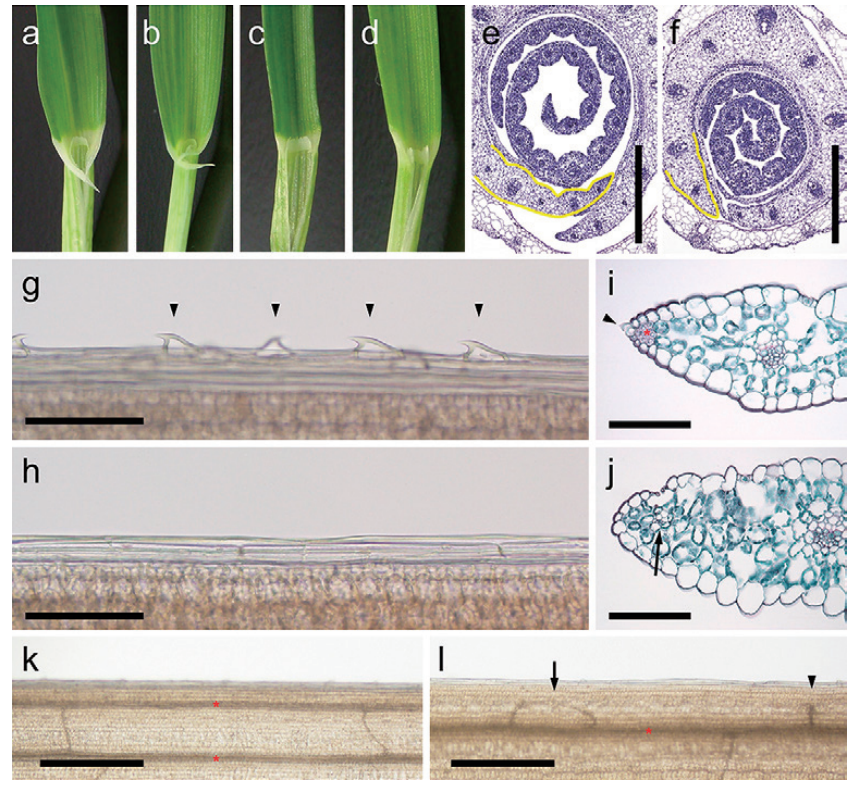

Fig. 3. Leaf marginal phenotypes in wild-type and nld1. (a-d) Closeup of the lamina-joint of the 1st leaf (a, c) and the 2nd leaf (b, d) in wild-type (KN29) (a, b) and nld1.b (c, d). (e and f) Cross sections of the leaf primordia in wild-type (KN29) (e) and nld1.b (f). Leaf margins are outlined in yellow. ( $g$ and $h$ ) The epidermal cells in the leaf margins of the 2nd leaf-blade in wild-type (KN29) (g) and nld1.b (h). Arrow heads indicate the sawtooth hairs at the leaf margin. ( $i$ and $j$ ) Cross sections of the leaf margins of the 2nd leaf-blades in wild-type (KN29) (i) and $n l d 1 . b(\mathrm{j})$. The sections are double-stained in safranin and fast green. The arrow head and asterisk in (i) indicate the sawtooth hair and sclerenchymatous cells in the leaf margin of wild-type, respectively. The arrow in (j) indicates the narrow vein in the leaf margin of nld1.b. (k and l) Vein patterns in the 2nd leaf-blades in wild-type (Nagaoka) (k) and nld1.a (1). The asterisks indicate longitudinal veins, and the arrow and arrow heads in (1) indicate the looped and interrupted commissural vein in the leaf margin of nld1.a, respectively. Bars $=500 \mu \mathrm{m}$ $(e, f, k, 1), 100 \mu \mathrm{m}(\mathrm{g}-\mathrm{j})$.

tants (Fig. 3i, 3j). The transparentization of leaf blades revealed that these marginal narrow veins are attributable to the looped commissural veins toward leaf margins (Fig. 3k, 31). The malformations of commissural veins, such as loop or interruption, were frequently observed in the leaf margin of nld1. These abnormal developments of commissural veins also indicate the abnormal development of leaf margins in the mutants.

The dwarf phenotype is another major characteristic of nld1 mutants (Fig. 4a). The comparison of the internode length indicated that the 1 st and 2 nd internode from the top showed the significant reduction in length (Fig. 4b). Occasionally, internodes exhibit abnormal winding development (Fig. 4c, 4d). This trend was more prominent in the upper internode. The cross section of the internode revealed the malformed development in $n l d 1$; the region where leaf margins are adjacent showed ill development in the internode. It was, therefore, considered that impaired development of internode is related to the defective development of leaf margins. 

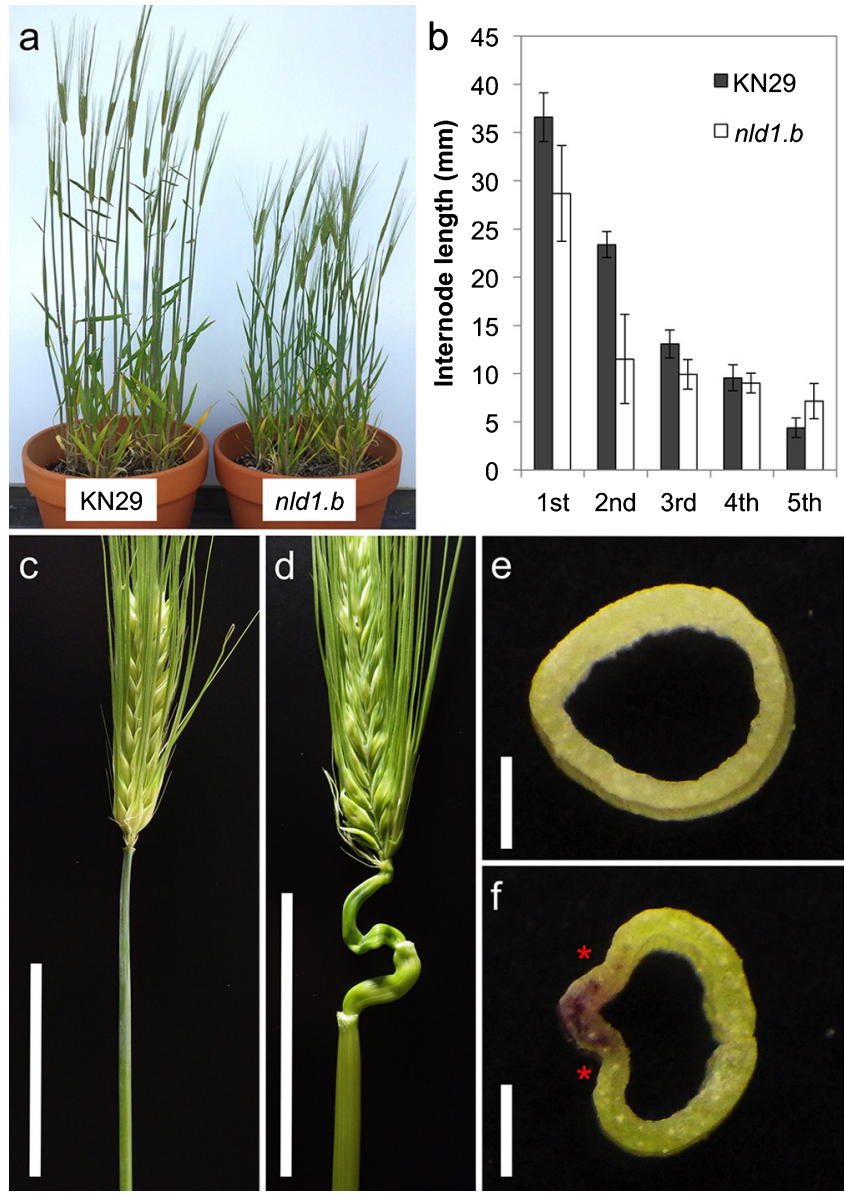

Fig. 4. Comparison of the internode development between wild-type and nldl mutants. (a) Matured plant phenotype of wild-type (KN29) and nld1.b. (b) Comparison of the internode length between wild-type (KN29) and nld1.b. Positions of internodes are indicated from the top. (c and d) The development of the internode in wild-type (Nagaoka) (c) and nld1.a (d). (e and f) The cross section of the 1st internode of wildtype (KN29) and nld1.b. The asterisks indicate the position to which leaf margins are adjacent. Bars $=5 \mathrm{~cm}(\mathrm{c}, \mathrm{d}), 1 \mathrm{~mm}(\mathrm{e}, \mathrm{f})$.

\section{Phenotypes of nld1 mutants in the reproductive phase}

The defective development of the marginal region is also appeared in the reproductive organs in the mutants. The lemmas, paleae, and empty glumes are apparently narrowed in $n l d 1$ (Fig. 5a). The histological analysis of spikelets revealed the marginal defect in lemmas and paleae in the mutants (Fig. 5b, 5c). The other organs such as stamens, pistils, and lodicules include no obvious malformations and the fertilities are comparable to those of the wild-types, suggesting that NLD1 is particularly involved in the development of lemmas and paleae in the reproductive organs. In matured spikes, the seeds are partly visible through gaps between lemmas and paleae in $n l d l$ because of incomplete overlap between margins of these organs (Fig. 5d, 5e). These results indicated that NLD1 play pivotal roles in the marginal development in vegetative and reproductive lateral organs.
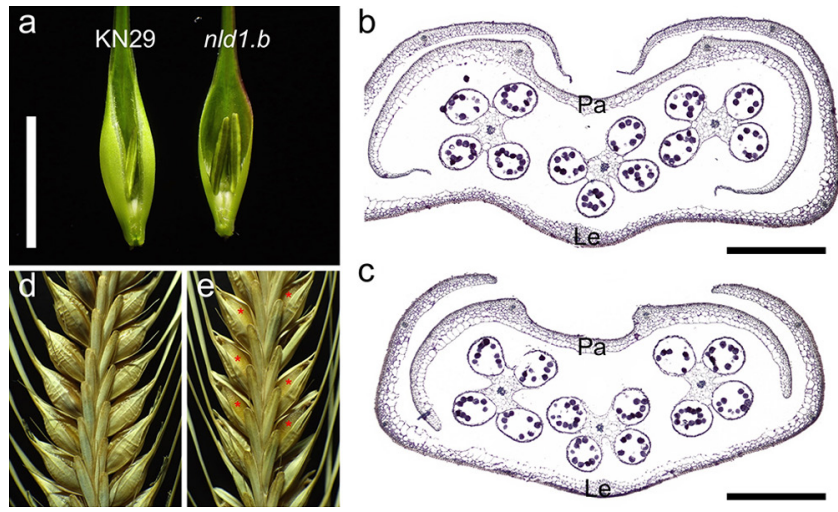

Fig. 5. Reproductive phenotypes in wild-type and nld1.b. (a) The spikelets of wild-type (KN29) and nld1.b. The paleae are removed so that the difference in lemma development are easy to be viewed. (b and c) The cross sections of spikelets in wild-type (KN29) (b) and $n l d 1 . b$ (c). The paleae (Pa) and lemmas (Le) are shown in the figure. (d and e) Matured inflorescence of wild-type (KN29) (d) and nld1.b (e). The asterisks indicate the enlarged gaps between paleae and lemmas in the mutant. Bars $=1 \mathrm{~cm}(\mathrm{a}), 200 \mu \mathrm{m}(\mathrm{b}, \mathrm{c})$.

\section{Map-based cloning of NLD1 gene}

The previous study reported that NLD1 is located about $21.6 \mathrm{cM}$ proximal from the fragile stem 1 locus on chromosome 5HL (Hayashi et al. 1983, Takahashi et al. 1972). The NLD1 locus was further limited within the region ranged from $76.4 \mathrm{cM}(\mathrm{k} 03390)$ to $80.7 \mathrm{cM}$ (k01939), which include WOX3 encoding MLOC_7772.1, an orthologous gene of maize NS1 and NS2 and rice NAL2/3. The ns1 ns2 and nal2/3 mutants exhibit narrowed leaves and spikelets due to the marginal defect, which seems to be similar to nldl phenotypes. Thus, we performed the sequence analysis of MLOC 7772.1 in nld1 mutants and wild-types, and found mutant specific alterations in both nld1.a and nld1.b (Fig. 6a). It was revealed that nld1.a contained point mutation from $\mathrm{G}$ to $\mathrm{A}$ at $740 \mathrm{bp}$, which cause nonsense mutation in the middle of amino acid sequence, and that $n l d 1 . b$ deleted one nucleotide at $275 \mathrm{bp}$, which cause drastic alteration in the amino acid sequence due to the frame shift mutation. WOX3 proteins can be classified into two clades; one consists of maize NS-related proteins and the other rice DEPILOUS (DEP)-related proteins (Fig. 6b). It is likely that barley possess two WOX3 genes ( $H v N S / N L D 1$ and HvWOX3), but HvWOX3 is classified into DEP-related clade. Therefore, it is likely that NS-related WOX3 gene is only $N L D 1 / H v N S$, which can account for the phenotypic alteration in nldl single mutant. The amino acid sequence alignment showed that NLD1-related proteins contain two conserved regions in N-terminal (the homeobox domain) and C-terminal (the WUSCHEL [WUS] box motif) (Fig. 6c). Both nld1.a and nld1.b possess intact homeobox domain but completely lost the WUS box motif (Fig. 6c). From these results, it was concluded that MLOC 7772.1 is identical to $N L D 1$. 
a

MLOC_7772.1 (NLD1/HvNS)

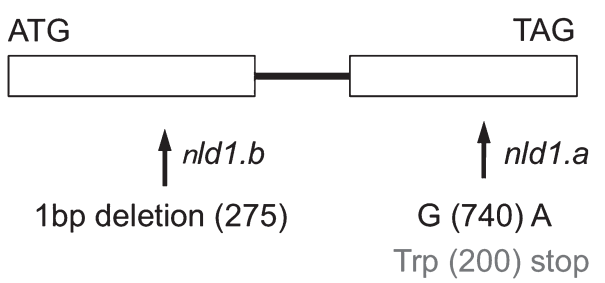

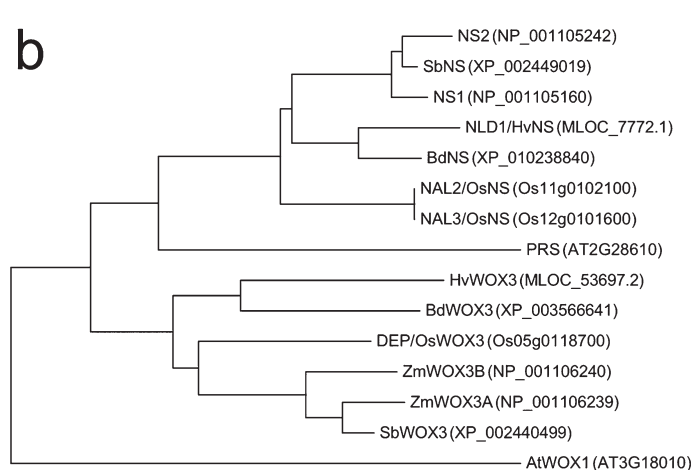

C

NS2 (NP 001105242) SbNS (XP 002449019) NS1 (NP 001105160) BdNS (XP 010238840) NAL2 (Os1190102100) NAL3 (Os12g0101600)

NS2 (NP 001105242) SbNS (XP 002449019) NS1 (NP 001105160)
NLD1 (MLOC 7772.1) NLD1 (MLOC 7772.1)
BdNS (XP 010238840) BdNS (XP 010238840)
NAL2 (Os 1190102100$)$ NAL2 (Os1190102100)

NS2 (NP 001105242) SbNS (XP 002449019) NS1 (NP 001105160) NLD1 (MLOC 7772.1) NAL2 (Os1190102100) NAL3 (Os12g0101600)

NS2 (NP 001105242) SDNS (XP 002449019) NS1 (NP 001105160) NLD1 (MLOC 7772.1)
BdNS (XP 010238840) BdNS (XP 010238840)
NAL2 (Os1190102100) NAL2 (Os11g0102100)
NAL3 (Os12g0101600)

NS2 (NP 001105242) SbNS (XP 002449019) NS1 (NP 001105160$)$ BdNS (XP 010238840) NAL2 (Os1190102100) NAL3 (Os1290101600)
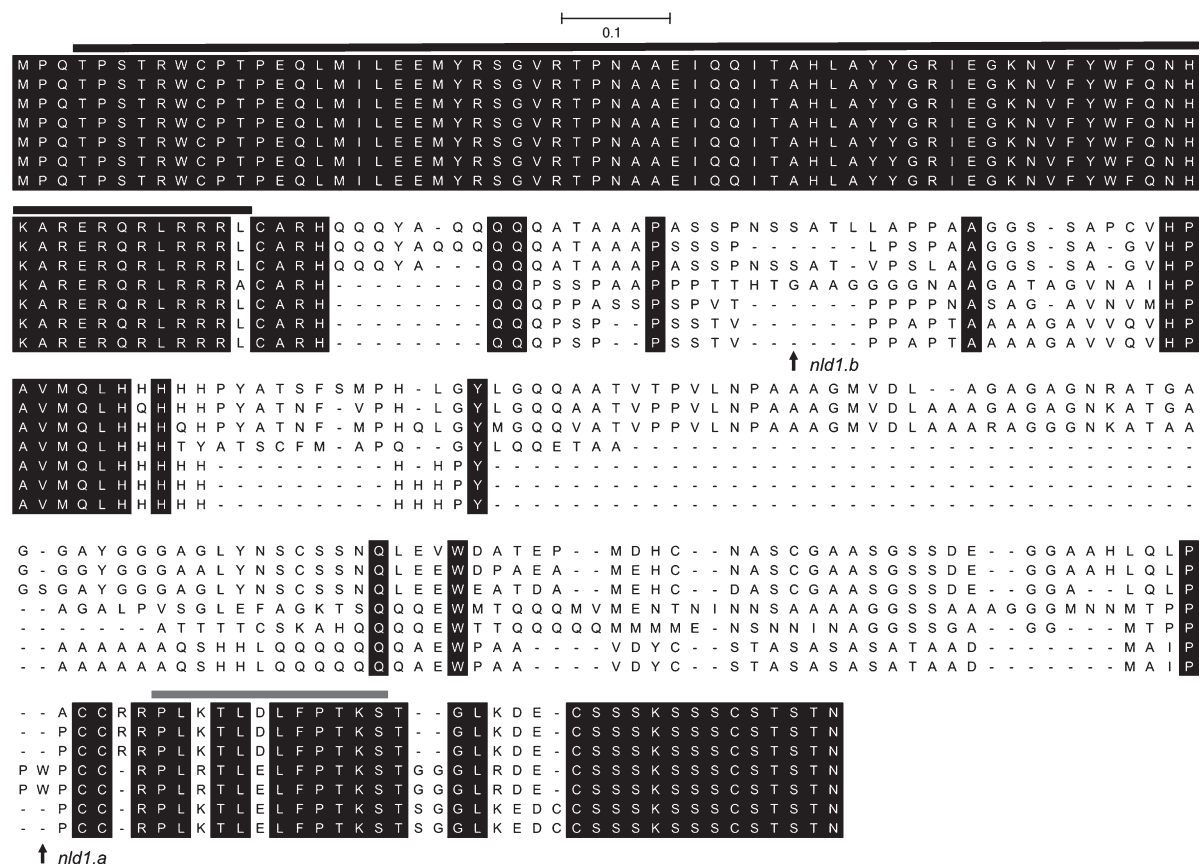

Fig. 6. Molecular characterization of the NLD1 gene. (a) Exon-intron structure and the mutation sites of $n l d 1 . a$ and $n l d 1 . b$. Exons and intron are shown by boxes and bold line, respectively. (b) Phylogenetic tree of NLD1-related proteins in barley (NLD1; MLOC_7772.1, HvWOX3; MLOC 53697.2), maize (NS1; NP 001105160, NS2; NP 001105242, ZmWOX3A; NP 001106239, ZmWOX3B; NP 001106240), rice (NAL2; Os11g0102100, NAL3; Os12g0101600, DEP; Os05g0118700), sorghum (SbNS; XP 002449019, SbWOX3; XP 002440499), Brachypodium distachyon (BdNS; XP_010238840, BdWOX3; XP_003566641), and Arabidopsis thaliana (PRS; AT2G28610, AtWOX1; AT3G18010). The tree was created using MEGA ver. 5.2.2 (available at http://megasoftware.net/, Tamura et al. 2011). (c) Comparison of the amino acid sequences among NLD1-related proteins in the NS cluster. Amino acid sequences were aligned with MUSCLE program of MEGA ver.5.2.2. Amino acids conserved in all proteins are represented by black boxes. Mutation sites in $n l d 1 . a$ and nld1.b are indicated with arrows. Black line and gray line indicate the homeobox domain and the WUS box motif, respectively.

\section{Expression analysis of NLD1 gene}

For the further understanding of the function of NLD1 in barley development, we performed expression analysis of $N L D 1$ by in situ hybridization. The earliest expression in the course of leaf development was observed in the marginal edges of the initiating leaf ridge (Fig. 7a, 7b). These marginal expression were continually observed throughout the development of leaf ridge and leaf primordium, and were kept as late as P4 leaf primordium (Fig. 7c). These expression pattern indicate that $N L D 1$ plays pivotal role not only in the leaf-ridge generation from SAM but also in the development of leaf margins. Other than leaf edges, NLDI transcripts were also observed on the epidermal cells along with the longitudinal veins in leaf primordia later than P3 stage (Fig. 7c). While NLD1 transcripts were continuously localized in leaf edges, those on the epidermal cells along with the longitudinal vein is interspersed (Supplemental Fig. 5), suggesting that $N L D 1$ plays different roles between these places. In reproductive organs, $N L D 1$ expression was observed in the marginal edges of lemmas, paleae, and empty glumes (Fig. 7d, 7e), which corresponded with the phenotypic alteration in nld 1 mutants (Fig. 5).

From these results, we concluded that $N L D 1$ plays pivotal roles in the increase of organ width and in the development of marginal tissues by expressing in the marginal edge of lateral organs in barley. 


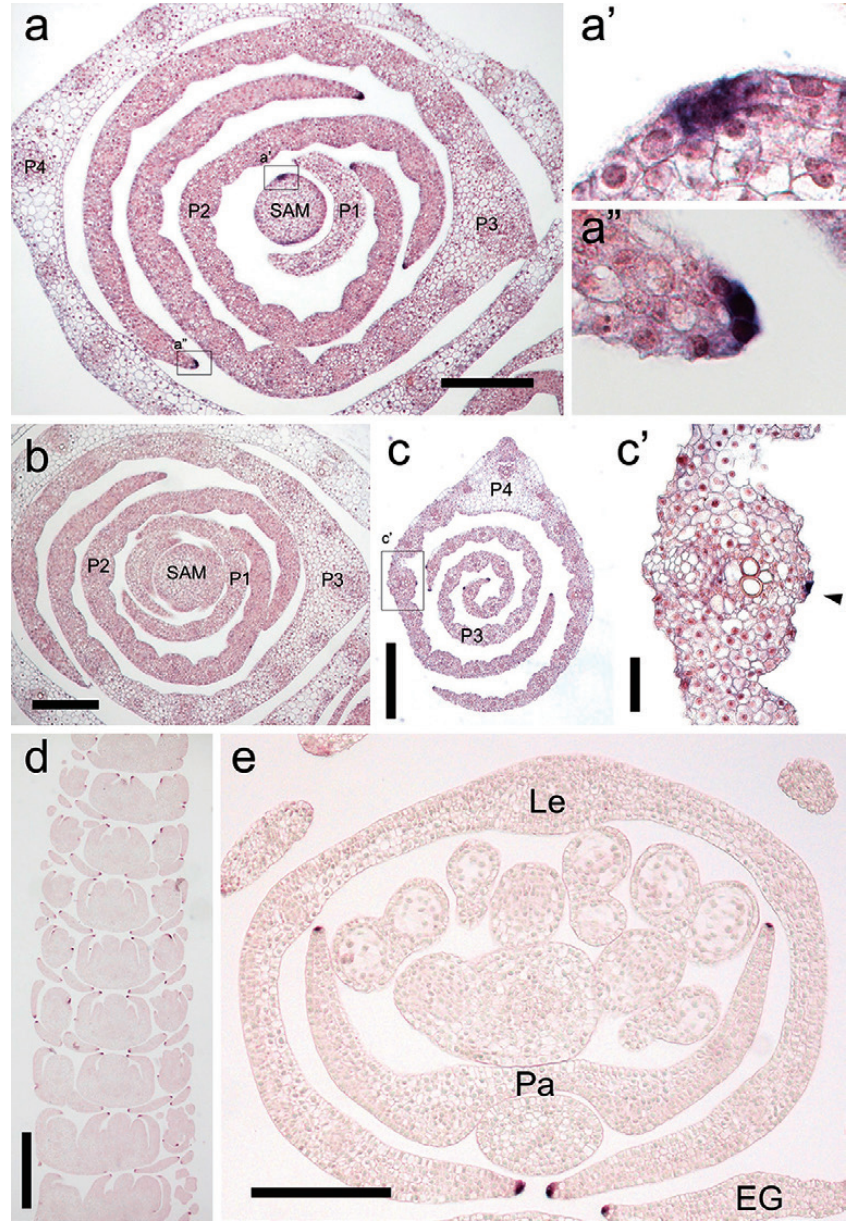

Fig. 7. Expression pattern of the $N L D 1$ gene. (a and b) Cross sections of shoot apex in wild-type (KN29) hybridized with $N L D 1$ anti-sense probe (a) and sense probe (b). In (a), parts of the section enclosed with the rectangles are enlarged in $\left(a^{\prime}\right)$ and $\left(a^{\prime \prime}\right)$. Seedlings at the 2nd leaf stage are used as the plant materials. Shoot apical meristem (SAM) and leaf primordial stages (P1-P4) are shown in the figures. (c) Cross section of leaf primordia in wild-type (KN29) hybridized with NLD1 anti-sense probe. Part of the section enclosed with the rectangle is enlarged in $\left(c^{\prime}\right)$. Seedling at the 2nd leaf stage is used as the plant material. Leaf primordial stages (P3 and P4) are shown in the figure. Arrow head in $\left(c^{\prime}\right)$ indicates the signal on the leaf epidermal cell. ( $d$ and e) Longitudinal section (d) and cross section (e) of the immature spikelets in wild-type (KN29) hybridized with $N L D 1$ anti-sense probe. The paleae $(\mathrm{Pa})$, lemmas (Le), and empty glume (EG) are shown in the figure. $\operatorname{Bars}=200 \mu \mathrm{m}$ (a, b, e), $400 \mu \mathrm{m}$ (c), $50 \mu \mathrm{m}\left(\mathrm{c}^{\prime}\right), 500 \mu \mathrm{m}(\mathrm{d})$.

\section{Discussion}

In the present study, we showed that the narrowed leaf phenotype of nld 1 mutants were attributable to the lack of the marginal regions in the leaves (Fig. 3g-3j). The defective development of margins are derived from the impaired development of leaf primordia (Fig. 2, Fig. 3e, 3f). Map-based cloning revealed that $N L D 1$ encodes a NS-related WOX3 protein, whose expression was localized in the marginal edges of lateral organs (Fig. 7). From these results, we con- cluded that NLD1 plays pivotal role in the increase of organ width and in the development of marginal tissues in lateral organs.

Maize ns 1 ns 2 mutants and rice nal2/3 mutants exhibit significant reduction in the width of leaves and floral organs due to the defective marginal development (Cho et al. 2013, Ishiwata et al. 2013, Nardmann et al. 2004, Scanlon et al. 1996, Scanlon and Freeling 1998). In $n s 1$ ns2, additional features such as stem curvature and shortened internodes are appeared, which seem to be attributable to the uneven internode growth (Scanlon et al. 1996). These mutant phenotypes resemble those of $n l d 1$ mutants. In addition, the transcripts of $N S$ and $N A L 2 / 3$ genes are also accumulated to the marginal edges of lateral organs (Nardmann et al. 2004, 2007). These similarities in loss-of-function mutant phenotypes and gene expression patterns strongly indicate that molecular function of $N S$-related WOX3 genes is highly conserved across plant species.

The earliest NLDI expression in the course of leaf development was observed in the marginal edges of the initiating leaf ridge (Fig. 7a). Maize $N S$ genes are suggested to be involved in the recruitment of leaf founder-cells by downregulating KNOX accumulation in the pre-marginal regions (Scanlon 2000, Scanlon and Freeling 1997, Scanlon et al. 2000). Thus, it is conceivable that $N L D 1$ also plays critical roles in the recruitment of leaf founder-cells. However, NLD1 transcripts were continually observed throughout the development of leaf primordium until as late as P4 stage (Supplemental Fig. 4). The expression of $N L D 1$ in such a late primordial stage indicates that $N L D 1$ also play crutial roles in the establishment and maintenance of marginal regions. Leaf margin functions as an adaxial-abaxial boundary, where adaxial and abaxial regulators are down-regulated by WOX genes (Nakada and Okada 2013). This is well demonstrated by woxl prs double mutants, which show confused adaxial-abaxial identity at leaf margins (Nakata et al. 2012, Vandenbussche et al. 2009). The lack of leafmargin specific structures and the thickening of leaf margins in $n l d 1$ may indicate that leaf adaxial-abaxial boundary is compromised in the mutants (Fig. 3g-3j).

Other than leaf edges, $N L D 1$ transcripts were also observed on the epidermal cells along with the longitudinal veins in leaf primordia later than P3 stage (Fig. 7c). Since such expressions have never been reported in other plants, this might be unique to barley. The signals were interspersed on the epidermal cells along with the longitudinal vein (Supplemental Fig. 5), which seem to be similar pattern to the development of the trichomes. In fact, nld 1 show impaired trichome development such as the reduction in trichome density and size (Supplemental Fig. 4), although such phenotypes have never been reported in other plants. Further study is required to reveal the role of $N L D 1$ gene in the development of leaf epidermal cells.

The development of leaf margin was impaired in nldl mutants (Fig. 3e, 3f). Occasionally, nld1 shows asymmetrical development of the leaf in medial-lateral directions 
(Supplemental Fig. 3). These mutant phenotypes suggest that NLD1 promote the expansion of lamina in the development of leaf primordium. However, the NLD1 transcripts were strictly limited within the few cells in leaf edges, implying that NLD1 functions non-cell-autonomously. This idea is supported by the lack of sclerenchymatous cells in nld1 locating inner than epidermal layer, where NLD1 expresssion was not observed (Fig. 3i, 3j), or by the malformation of commissural veins in the leaf margin of the mutants (Fig. 3k, 3l). This contradiction could be explained by the migration of either NLD1 protein itself or the secondary signals derived from the marginal cells. Previous studies have shown that polar auxin transport plays an important role in determining vascular pattern in leaves (Sakaguchi et al. 2010, Scarpella et al. 2006), and loss-of-funcion of auxin biosynthesis or transport genes gave rise to reduction in leaf blades in rice (Fujino et al. 2008, Qi et al. 2008, Yoshikawa et al. 2014). Therefore, it is conceivable that $n l d l$ includes some abnormalities in auxin transport. Cho et al. (2013) also referred to the possibility that nal2/3 phenotypes are partly attributable to the altered auxin transport. Thus, it is quite interesting whether auxin functions as the secondary signal of $N S$-related $W O X 3$ genes.

In the present study, we identified barley NLD1 gene, which encodes NS-related WOX3 protein (HvNS). Database analysis revealed that barley possess another WOX3 gene $(H v W O X 3)$, which showed higher similarity to rice $D E P$ gene than to $N L D 1 / H v N S$, suggesting that these two genes were differentiated earlier than the differentiation of rice and barley. While NS-related WOX3 genes have been widely studied, the information about DEP-related WOX3 genes is still limited (Angeles-Shim et al. 2012). Thus, further study of $N L D 1 / H v N S$ and $H v W O X 3$ will not only reveal the molecular mechanism of barley development but also provide new insight into the evolution of WOX3 gene families in plants.

\section{Acknowledgements}

Nagaoka-dwarf (nld.1a) mutant and its wild-type was supplied by under the support of National Bio Resource Project-Barley, Japan. The authors are grateful for Dr. T. Sootome, for generous supply of $n l d 1 . b$ induced from Kanto 29. This study was supported in part by the Joint Research Program of the Institute of Plant Science and Resources, Okayama University.

\section{Literature Cited}

Aloni, R., K. Schwalm, M.Langhans and C.I. Ullrich (2003) Gradual shifts in sites of free-auxin production during leaf-primordium development and their role in vascular differentiation and leaf morphogenesis in Arabidopsis. Planta 216: 841-853.

Angeles-Shim, R.B., K.Asano, T.Takashi， J.Shim, T.Kuroha, M.Ayano and M.Ashikari (2012) A WUSCHEL-related homeobox 3B gene, depilous (dep), confers glabrousness of rice leaves and glumes. Rice 5: 28 .

Barkoulas, M., A.Hay, E.Kougioumoutzi and M.Tsiantis (2008) A developmental framework for dissected leaf formation in the Arabidopsis relative Cardamine hirsuta. Nat. Genet. 40: 1136-1141.

Benjamins, R. and B. Scheres (2008) Auxin: the looping star in plant development. Annu. Rev. Plant Biol. 59: 443-465.

Bilsborough, G.D., A. Runions, M. Barkoulas, H.W. Jenkins, A. Hasson, C. Galinha, P.Laufs, A.Hay, P. Prusinkiewicz and M.Tsiantis (2011) Model for the regulation of Arabidopsis thaliana leaf margin development. Proc. Natl. Acad. Sci. USA 108: 3424-3429.

Cho, S.H., S.C.Yoo, H.Zhang, D.Pandeya, H.J.Koh, J.Y.Hwang, G.T. Kim and N.C.Paek (2013) The rice narrow leaf2 and narrow leaf3 loci encode WUSCHEL-related homeobox 3A (OsWOX3A) and function in leaf, spikelet, tiller and lateral root development. New Phytol. 198: 1071-1084.

Fujino, K., Y.Matsuda, K. Ozawa, T.Nishimura, T.Koshiba, M.W. Fraaije and H. Sekiguchi (2008) NARROW LEAF 7 controls leaf shape mediated by auxin in rice. Mol. Genet. Genomics 279: 499507.

Giacomo, E.D., M.A. Iannelli and G. Frugis (2013) TALE and shape: How to make a leaf different. Plants 2: 317-342.

Haecker,A., R. Gross-Hardt, B. Geiges, A. Sarkar, H. Breuninger, M.Herrmann and T.Laux (2004) Expression dynamics of WOX genes mark cell fate decisions during early embryonic patterning in Arabidopsis thaliana. Development 131: 657-668.

Hay, A., J. Craft and M. Tsiantis (2004) Plant hormones and homeoboxes: bringing the gap? BioEssays 26: 395-404.

Hay,A., M.Barkoulas and M.Tsiantis (2006) ASYMMETRIC LEAVES1 and auxin activities converge to repress BREVIPEDICELLUS expression and promote leaf development in Arabidopsis. Development 133: 3955-3961.

Hayashi, J., I. Moriya and R. Takahashi (1983) A further study on linkage of the "dusky" mutant, dsk. BGN 13: 42-44.

Heisler, M.G., C.Ohno, P.Das, P. Sieber, G.V.Reddy, J.A. Long and E.M.Meyerowitz (2005) Patterns of auxin transport and gene expression during primordium development revealed by live imaging of the Arabidopsis inflorescence meristem. Curr. Biol. 15: 1899-1911.

Ishiwata,A., M. Ozawa, H. Nagasaki, M. Kato, Y.Noda, T. Yamaguchi, M.Nosaka, S.Shimizu-Sato, A.Nagasaki, M. Maekawa et al. (2013) Two WUSCHEL-related homeobox genes, narrow leaf2 and narrow leaf3, control leaf width in rice. Plant Cell Physiol. 54: 779-792.

Kouchi, H. and S. Hata (1993) Isolation and characterization of novel nodulin cDNAs representing genes expressed at early stages of soybean nodule development. Mol. Gen. Genet. 238: 106-119.

Matsumoto, N. and K. Okada (2001) A homeobox gene, PRESSED FLOWER, regulates lateral axis-dependent development of Arabidopsis flowers. Genes Dev. 15: 3355-3364.

McHale, N.A. and M. Marcotrigiano (1998) LAM1 is required for dorsoventrality and lateral growth of the leaf blade in Nicotiana. Development 125: 4235-4243.

Moon, J. and S. Hake (2011) How a leaf gets its shape. Curr. Opin. Plant Biol. 14: 24-30.

Nakata, M., N. Matsumoto, R. Tsugeki, E. Rikirsch, T.Laux and K. Okada (2012) Roles of the middle domain-specific WUSCHELRELATED HOMEOBOX genes in early development of leaves in Arabidopsis. Plant Cell 24: 519-535.

Nakata, M. and K. Okada (2013) The leaf adaxial-abaxial boundary and lamina growth. Plants 2: 174-202.

Nardmann, J., J. Ji, W. Werr and M.J. Scanlon (2004) The maize duplicate genes narrow sheath1 and narrow sheath 2 encode a conserved 
homeobox gene function in a lateral domain of shoot apical meristems. Development 131: 2827-2839.

Nardmann, J., R.Zimmermann, D. Durantini, E. Kranz and W.Werr (2007) WOX gene phylogeny in Poaceae: A comparative approach addressing leaf and embryo development. Mol. Biol. Evol. 24: 2474-2484.

Petrášek, J. and J.Friml (2009) Auxin transport routes in plant development. Development 136: 2675-2688.

Qi, J., Q. Qian, Q.Bu, S.Li, Q.Chen, J. Sun, W.Liang, Y.Zhou, C. Chu, X.Li et al. (2008) Mutation of the rice Narrow leafl gene, which encodes a novel protein, affects vein patterning and polar auxin transport. Plant Physiol. 147: 1947-1959.

Sakaguchi, J., J.Itoh, A.Nakamura, H.Fukuda and S.Sawa (2010) COE1, an LRR-RLK responsible for commissural vein pattern formation in rice. Plant J. 63: 405-416.

Sarojam, R., P.G. Sappl, A. Goldshmidt, I.Efroni, S.K. Floyd, Y.Eshed and J.L.Bowman (2010) Differentiating Arabidopsis shoots from leaves by combined YABBY activities. Plant Cell 22: 2113-2130.

Sato, K., N. Nankaku and K. Takeda (2009) A high-density transcript linkage map of barley derived from a single population. Heredity (Edinb) 103: 110-117.

Scanlon, M.J., R.G. Schneeberger and M.Freeling (1996) The maize mutant narrow sheath fails to establish leaf margin identity in a meristematic domain. Development 122: 1683-1691.

Scanlon, M.J. and M. Freeling (1997) Clonal sectors reveal that a specific meristematic domain is not utilized in the maize mutant narrow sheath. Dev. Biol. 182: 52-66.

Scanlon, M.J. and M. Freeling (1998) The narrow sheath leaf domain deletion: a genetic tool used to reveal developmental homologies among modified maize organs. Plant J. 13: 547-561.

Scanlon, M.J. (2000) NARROW SHEATH1 functions from two meristematic foci during founder-cell recruitment in maize leaf development. Development 127: 4573-4585.

Scanlon, M.J., K.D. Chen and C.C.McKnightIV (2000) The narrow sheath duplicate genes: sectors of dual aneuploidy reveal ancestrally conserved gene functions during maize leaf development. Genetics 155: 1379-1389.

Scarpella, E., D. Marcos, J. Friml and T. Berleth (2006) Control of leaf vascular patterning by polar auxin transport. Genes Dev. 20: 1015-
1027.

Scarpella,E., M. Barkoulas and M. Tsiantis (2010) Control of leaf and vein development by auxin. Cold Spring Harb. Perspect. Biol. 2: a001511.

Shewry, P.R. (1992) Barley: genetics, biochemistry, molecular biology and biotechnology. Oxford University Press, pp. 231-263.

Stahle, M.I., J.Kuehlich, L.Staron, A.G.vonArnim and J.F.Golz (2009) YABBYs and the transcriptional corepressors LEUNIG and LEUNIG_HOMOLOG maintain leaf polarity and meristem activity in Arabidopsis. Plant Cell 21: 3105-3118.

Takahashi, R., J.Hayashi, T. Konishi and I. Moriya (1972) Inheritance and linkage studies in barley. V. Locating of seven new mutant genes. Ber. Ohara Inst. landw. Biol., Okayama Univ. 15: 147-168.

Tadege, M., H.Lin, M.Bedair, A.Berbel, J.Wen, C.M. Rojas, L.Niu, Y.Tang, L. Sumner, P. Ratet et al. (2011) STENOFOLIA regulates blade outgrowth and leaf vascular patterning in Medicago truncatula and Nicotiana sylvestris. Plant Cell 23: 2125-2142.

Tamura, K., D. Peterson, N. Peterson, G. Stecher, M. Nei and S. Kumar (2011) MEGA5: Molecular Evolutionary Genetics Analysis using maximum likelihood, evolutionary distance, and maximum parsimony methods. Mol. Biol. Evol. 28: 2731-2739.

Vandenbussche, M., A.Horstman, J.Zethof, R.Koes, A.S. Rijpkema and T. Gerats (2009) Differential recruitment of WOX transcription factors for lateral development and organ fusion in Petunia and Arabidopsis. Plant Cell 21: 2269-2283.

Varshney, R.K., T.C.Marcel， L.Ramsay， J.Russell， M.S.Röder, N.Stein, R. Waugh, P. Langridge, R.E. Niks and A. Graner (2007) A high density barley microsatellite consensus map with 775 SSR loci. Theor. Appl. Genet. 114: 1091-1103.

Vernoux, T., F. Besnard and J. Traas (2010) Auxin at the shoot apical meristem. Cold Spring Harb. Perspect. Biol. 2: a001487.

Wenzel, C.L., M.Schuetz, Q.Yu and J.Mattsson (2007) Dynamics of MONOPTEROS and PIN-FORMED1 expression during leaf vein pattern formation in Arabidopsis thaliana. Plant J. 49: 387-398.

Yoshikawa, T., M.Ito, T. Sumikura, A. Nakayama, T.Nishimura, H.Kitano, I.Yamaguchi, T.Koshiba, K. Hibara, Y. Nagato et al. (2014) The rice FISH BONE gene encodes a tryptophan aminotransferase, which affects pleiotropic auxin-related processes. Plant J. 78: 927-936. 